\title{
Changes in straight-ahead eye position during adaptation to wedge prisms
}

SAMUEL C. MGLAUGHLIN AND ROBERT G. WEBSTER

VISUAL PERCEPTION LABORATORY, TUFTS UNIVERSITY

\begin{abstract}
If $S$ is instructed to look straight ahead before adapting
\end{abstract} to laterally displaced vision, he does so without noticeable error. After adapting, however, in response to the same instruction, he may rotate his eyes as much as $8^{\circ}$ toward the the displaced visual target. This is the change in judgment of the direction of gaze which Helmholtz identified in 1867 as an important physiological mechanism in adaptation to prisms. It leads to more accurate reaching behavior by causing $S$ to make a visual judgment that the target is closer to straight ahead than it was when he first looked through the prisms. This type of adaptive change (change in judgment of the direction of gaze, oculomotor change) can be measured either by manual judgments (difference between successive "straight ahead" and "visual target' judgments) or by changes in straight-ahead eye position. It may be described as a parametric adjustment in the oculomotor control system, and is closely analogous to the eye movement which subserves the recovery of binocular fusion in prism vergence.

Adaptation to laterally displacing wedge prisms was described by von Helmholtz in 1867. Wearing spectacles which contained the prisms, he first demonstrated the apparent displacement by looking at objects, the closing his eyes and trying to touch them. He reached incorrectly, the direction of his error being determined by the orientation of the prisms. He then found that he could eliminate the error in either of two ways: by reaching repeatedly for objects with his eyes closed, or, "more quickly still," by touching the objects several times while watching his hand through the prisms. Having adapted by one or the other of these techniques, he repeated the initial procedure as a means of demonstrating that adaptation had taken place: "on trying the above experiment again, we shall discover that now we do not miss the objects but feel for them correctly." 1

Helmholtz went on to investigate the locus of the adaptive effect. He found that, if he adapted by doing all of the reaching with his right hand, keeping his left hand out of his field of view, the adaptive effect nevertheless transferred fully to his left hand. He concluded that the adaptive change could not be in his judgment of the position of his hand, but must be in the "judgment of the direction of the gaze."

That brief, century-old account contains a great deal of insight into the physiological basis of prism adaptation. Helmholtz recognized that, from a physiological point of view, there were two acceptable hypotheses which might account for the improved accuracy of reaching behavior, and only two. There might be a change in the judged position of the hand seen through prisms, so that during the test procedure (looking at an object, then closing one's eyes and trying to touch it), one tried to reach incorrectly but reached correctly. Or, there might be a change in the judgment of the direction of gaze, so that, when one looked at the (optically displaced) object, one felt oneself to be looking straight ahead. This, too, could lead to accurate reaching behavior, for, having identified the object with visual straight ahead, one would reach straight ahead in attempting to touch it. Helmholtz' test for intermanual transfer, apparently with only himself as subject, enabled him to make a quick choice between these two alternatives.

A change in the judgment of the direction of gaze may be manifested in any one of a number of ways. While judging himself to be looking straight ahead, $s$ may turn his eyes in his head, or he may turn his head while holding his eyes straight ahead in their sockets, or he may turn his head and trunk, and so on. In the present investigation, we are concerned specifically with the eye-turning response. One reason for this choice of emphasis is that it is easier to eliminate head and trunk movements than it is to eliminate eye movements. Another reason is that ocular responses have been investigated far more extensively than have head and trunk turning responses, so that there is more opportunity to relate ocular findings to the work of investigators in fields other than prism adaptation.

Each of the two adaptive mechanisms which is implied in Helmholtz' experiment involves a change in the judged position of a part of the body. However, in the present paper, the term "proprioceptive change" will be reserved for a change in the judged position of a part of the body seen through prisms; and the term "oculomotor change" will be used to designate a change in the judgment of the direction of gaze as manifested by a change in straight-ahead eye position.

Helmholtz' finding of lack of proprioceptive change is evidently not general, for there is now a substantial body of evidence that proprioceptive changes do occur during adaptation. The nature of the adaptive change appears to be complexly related to the conditions of adaptive exposure; and it is not uncommon for the two kinds of change-proprioceptive and oculomotorto occur simultaneously, combining additively to produce the total adaptive effect (Harris, 1963, 1965; Hamilton, 1964; McLaughlin \& Bower, 1965; Hay \& Pick, 1966; McLaughlin, Rifkin, \& Webster, 1966). 
So far as we have been able to determine, there has been only one previous prism-adaptation experiment in which measures of eye position were reported (Kalil \& Freedman, 1966). In that experiment, however, no other measures were made except measures of eye position, so that there is no way to correlate the observed oculomotor change with changes in reaching behavior, or even to be certain that there were any changes in reaching behavior. Moreover, measures were taken only "before" and "after" adaptation, both times with prisms removed. This technique of measurement provides no basis for estimating the amount of adaptive change that occurred during adaptation-i.e., while the prisms were in place. Some Ssparticularly those who are well-practiced in adapting to prisms-may show little or no after-effect following removal of the prisms, despite the fact that they had been fully adapted when the prisms were in place (Taylor, 1962).

\section{METHOD}

In the present experiment, $S$ 's judgment of the direction of gaze was evaluated by measuring his eye position while he attempted to look straight ahead, these measures being taken repeatedly during the course of adaptation. Measurements of reaching behavior were also made at frequent intervals during adaptation.

If there is a proprioceptive change associated with the hand and arm seen through the prisms, S will point inaccurately at any target, including straight ahead, so long as he does not see his hand while pointing (Harris, 1963; MeLaughlin \& Bower, 1965). Hence, proprioceptive changes can be measured by having $S$ point straight ahead with hand not seen. This technique was employed in the present experiment.

Subjects. Ss were 10 male college students.

Apparatus. The visual display consisted of a horizontal row of dots $8 \mathrm{~cm}$ below eye level on a vertical partition perpendicular to S's line of sight. Each dot was $3 \mathrm{~mm}$ in diameter, they were spaced $1 \mathrm{~cm}$ on center, and all were of approximately equal brightness. There were 69 of these dots, 34 on each side of a center dot. The dots were transilluminated, and the experimenter could turn them all on or all off, or he could turn on the center dot alone.

The $\mathrm{S}$ viewed this display from a distance of $37 \mathrm{~cm}$, his head being held in position by a bite board bearing his dental impression, and also by a chin rest. When making the dental impression, $S$ was instructed to position himself in such a way that the center dot (viewed binocularly) appeared to him to be straight ahead with respect to his head and body.

Directly beneath the row of dots was a sliding pointer which $\mathrm{S}$ could move along a horizontal track perpendicular to his line of sight. The pointer was $3 \mathrm{~cm}$ wide and contained a tactual reference mark (vertical line) $0.7 \mathrm{~mm}$ wide. The experimenter, on the other side of the horizontal partition, could read S's pointer settings and could move the pointer himself. All pointer settings were made with S's right hand.

The light from the transilluminated dots was not sufficient to enable $S$ to see his hand or the pointer; $S$ could see these only when $E$ illuminated them by means of special "side lights" installed on the inner side walls of the apparatus. With the side lights on, $S$ could see only his hand and the pointer, all other objects in the room being shielded from his view.

Throughout the experiment (after the dental impression had been made), S's left eye was occluded. The prism (20 diopters base right) was not in place at the start of the experiment, but was positioned before S's right eye by a remote control mechanism after $\mathrm{S}$ had made one pointer setting. This arrangement made it possible for $S$ to start the experiment without having seen the visual display through the prism, and to make one judgment (a "straight ahead" setting) without the prism. When the prism was in place, its center was approximately $34 \mathrm{~cm}$ from the row of dots and $3 \mathrm{~cm}$ from the front surface of S's cornea.

By means of an optical bench setup which reproduced the viewing distances and angles of incidence of the experimental situation, it was determined empirically that the prismatic displacement of the center dot as seen from S's right eye was $6.8 \mathrm{~cm}$ toward S's left.

The S's right eye was photographed through an aperture above the row of dots in the vertical partition. The location of the aperture was camouflaged so that it would not provide $S$ with a clue (accurate or inaccurate) to "straight ahead." A $35 \mathrm{~mm}$ camera was positioned so that its focal plane was approximately $75 \mathrm{~cm}$ from the eye. The eye was imaged on the film (Kodak High-Speed Infra-red) by a telephoto lens having a focal length of $18 \mathrm{~cm}$, positioned with its center approximately $46 \mathrm{~cm}$ from the eye. A small projector, directed toward the distal portion of the limbus of the eye (the border between iris and sclera), provided invisible infra-red radiation for photography. The source of radiation was an incandescent lamp, and the infra-red filter was a Wratten $87 \mathrm{c}$. The exposure time was .125 sec.

Procedure. Prior to the experiment, $S$ was shown how to position himself on the bite board and how to operate the pointer; but he was given no opportunity to look through the prism. He was also shown the camera, and was told that his eye would be photographed from time to time as a means of determining its position. He was instructed that all "straight ahead" settings were to be made with reference to his head and body and not with reference to his right eye. Finally, he was instructed to signal the completion of each pointer setting by tapping the table with his left hand.

With $\mathrm{S}$ in position and in total darkness, $E$ positioned the pointer 8-12 cm to right of center, then instructed $S$ to take hold of it ("you will find the pointer to your right") and to set it straight ahead. When S signalled the completion of this setting, E recorded it and 
instructed $S$ to remove his hand from the pointer.

The experimenter then put the prism in place by remote control, turned on the center dot, and set the pointer $8-12 \mathrm{~cm}$ toward S's right. The $S$ was again instructed to set the pointer straight ahead; and, at S's signal, this second setting was recorded and S was instructed to remove his hand from the pointer.

The experimenter then instructed $S$ to fixate the dot which appeared to him to be straight ahead with respect to his head and body, and to signal (by tapping the table) when he was doing so. At S's signal, the E turned on the infra-red projector, operated the camera, and turned the projector off.

Next, $E$ turned off all dots for about three seconds. The center dot was then turned on and $S$ was instructed to set the pointer directly beneath the dot and to keep his hand on the pointer when he had done so. At S's signal, E simultaneously recorded this setting, turned on the side lights, and repeated the instruction, "set the pointer directly beneath the dot." This setting was considered to have been completed only when it was in error by $1 \mathrm{~mm}$ or less, and when $\mathrm{S}$ had signalled.

The last four judgments ("straight ahead" setting with all dots on, eye photograph with all dots on, "visual target" setting in the dark with center dot on, and "visual target" setting with side lights and center dot on) were repeated 14 additional times without interruption. The initial straight ahead setting in total darkness was not repeated.

Calibration photographs. Immediately following the 15 prism-adaptation trials, with the prism still in place, with the side lights on, and with S's hand off the pointer, $E$ positioned the pointer directly beneath the dot that was $8 \mathrm{~cm}$ toward S's right from the center dot. The S was then instructed to fixate the dot above the pointer. When $\mathrm{S}$ signalled that he was doing so, a photograph was taken. This procedure was repeated with $\mathrm{S}$ fixating the dots at $6 \mathrm{~cm}$ right, $4 \mathrm{~cm}$ right, $2 \mathrm{~cm}$ right, center, and $2 \mathrm{~cm}$ left.

This sequence of six photographs was repeated four times.

Measurement of eye position. The $35 \mathrm{~mm}$ transparencies were projected to give overall magnification of about 30 (for photographic system, $M=0.65$; for projection system, $M=46$ ). The distance $d$ from an anatomical mark on the outer canthus to the nearest point on the limbus was measured on the projected image. The average measurements from the four sets of calibration photographs, plotted against the point of fixation on the horizontal row of dots, gave an empirical relationship between $\mathrm{d}$ and the point of fixation. The straight line describing this relationship (determined by visual inspection) was used in determining the point of fixation on each of the fifteen photographs taken during exposure to prism. Typically, the departure from linearity in this relationship was less than $0.1 \mathrm{~cm}$; the maximum departure from linearity for any $S$ at any point was $0.8 \mathrm{~cm}$.
To determine the reliability of this measurement procedure, calibration curves for six Ss were obtained twice, each time from the same set of photographs, and each time by the same $E$. The mean error between the first and second measurements was $15 \mathrm{~min}$. arc, and the standard deviation of the error distribution was $6.3 \mathrm{~min}$. arc.

\section{RESULTS}

The results of the experiment are summarized in Fig. 1, which shows the mean pointer settings and mean point of straight-ahead visual fixation for $10 \mathrm{Ss}$ in each of the 15 experimental trials.

Each of the three dependent variables plotted in Fig. 1 shows a shift in the expected direction. For manual settings straight ahead, the statistical significance of this shift was evaluated by comparing the mean of the first three trials with the mean of the last three trials. However, in the case of manual settings on the visual target, the initial setting (made prior to any adaptive exposure) was considerably in excess of the prism deviation. Since this large value would have biased the statistical analysis in such a way as to show significance where none acually existed, trial 1 was omitted and the mean of trials 2,3 , and 4 was compared with the mean of the last three trials. A similar procedure was followed in analysing the "visual fixation" measures. The results of these statistical analyses appear in Table 1.

The first manual setting on the visual target (mean for $10 \mathrm{Ss}$ ) differed from the prism deviation by $1.1 \mathrm{~cm}$. This difference is not statistically significant (C. R.= .384 , df $=9, p>.05$ ).

The first straight ahead visual fixation (mean for $10 \mathrm{Ss}$ ) differed from veridical straight ahead by $2.2 \mathrm{~cm}$. This difference is not statistically significant (C. R.= 1.90, df $=9, p>.05$ )

Figure 2 is a comparison between two variables, each derived from the data of Fig. 1. The first is $P F^{\prime}=6.8-P F$, where $P F$ is the point of visual fixation in Fig. 1. The second is the numerical difference between adjacent "straight ahead" and "visual target"

Tabie 1. Statistical summary of changes in manual settings and eye position during adaptation to displaced vision

\footnotetext{
1. Manual settings straight ahead

Mean of trials 2, 3, and 4: $0.56 \mathrm{~cm}$. (right)

Mean of trials 13, 14, and $15 ; 1.18 \mathrm{~cm}$. (right)

$t=1.56$, d.f. $=29, \mathrm{P}>.05$ (not significant)

2. Manual settings on visual target

Mean of settings 2, 3 , and 4: 3.45 (left)

Mean of settings 13, 14, and 15: 1.06 (left)

$t=6.68$, d.f. $=29, P<.01$

3. Visual fixation straight ahead

Mean of settings 2, 3 , and 4: 3.59 (left)

Mean of settings 13, 14, and 15: 2.67 (left)

$t=2.65$, d.f. $=29, P<.02$
} 


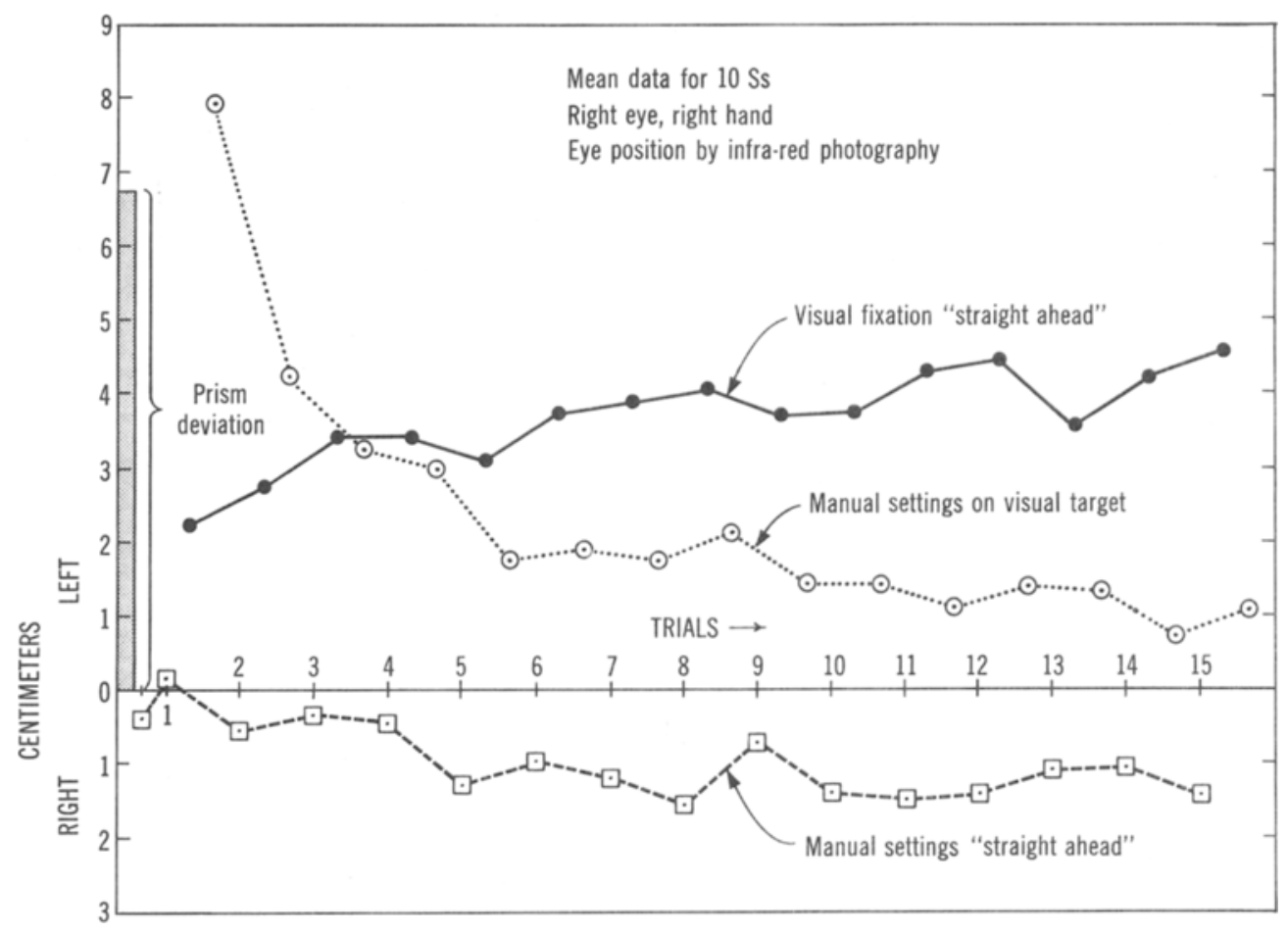

Fig. 1. Results of the experiment in terms of mean data for $10 \mathrm{Ss}$. Visual target was veridically straight ahead of $S$, and was displaced optically as indicated by the thickened portion of the ordinate. The terms "left" and "right" on the ordinal legend refer to S's left and right. All responses have been plotted in the order in which they occurred: thus, within each trial, the manual judgment of straight ahead precedes the eye position measure, which in tum precedes the manual setting on visual target.

settings. The purpose of the comparison is to show the similarity between the two, in support of the thesis that both are measures of the same thing-namely, change in judgment of the direction of gaze. Even with the large discrepancy between the two measures in trial 1 , the two are highly correlated $(r=.80, d f=14$, $p<.01)$ and not significantly different $\left(x^{2}=3.63\right.$, df $=$ $14, \mathrm{p}>.99)$.

\section{INTERPRETATION OF RESULTS}

Total change in reaching behavior. The shift in manual settings on the visual target (Fig. 1) represents the total change in reaching behavior during adaptation. Comparison of trial 1 with trial 15 shows this change to be $6.8 \mathrm{~cm}$. We consider this total change in reaching behavior to be the resultant of two components: proprioceptive and oculomotor.

Proprioceptive change. As indicated earlier, the shift in manual straight ahead judgments is the measure of proprioceptive change. Visual inspection of Fig. 1 suggests that there was such a shift, but Table 1 indicates that it was not statistically significant. For purposes of analyzing the total adaptive change into its components, it will be assumed that a proprioceptive shift did occur, and that its magnitude is represented by the difference between initial and terminal straight ahead judgments $(1.5 \mathrm{~cm})$.

Oculomotor change. There was a shift of $2.4 \mathrm{~cm}$ between initial and terminal points of straight ahead visual fixation. The direction of this shift was such that, as S adapted, his straight-ahead direction of gaze gradually shifted toward the visual target.

There are two things to be noted about this shift. First, it represents a shift in $S^{\top} S$ judgment of the direction of his gaze, for $S$ was following the same instruction ("look straight ahead") during all eye photographs. Second, in terms of S's visual experience, it is not a matter of visual straight ahead gradually becoming closer to the displaced target; it is a matter of the target becoming more nearly straight ahead. In other words, it is a change in the apparent position of the target. The variable FP' in Fig. 2 is intended to represent this change in S's visual experience.

The change in apparent position of the visual target also shows up in S's manual pointer settings. Every pair of settings ("straight ahead" and "visual target") requires $S$ to make a judgment as to how far from straight ahead the visual target appears to be-that is, how far it is "off to the side." It is on this basis that the numerical difference between adjacent straight ahead and visual target settings is taken as an index of oculomotor change in Fig. 2. 
A third independent measure of oculomotor change, in addition to those shown in Fig. 2, would be provided by S's verbal report of the apparent position of the target at each trial. This was not used in the present experiment, chiefly because of the difficulty of obtaining verbal reports with $S$ on a bite board.

As already noted, the point of straight ahead fixation in trial 1 differed from veridical straight ahead by $2.2 \mathrm{~cm}$. Even though this difference is not statistically significant, it is large enough to be an obstacle to the use of eye position data in calculating the proportion of total adaptive change which was oculomotor. For this reason, the manual pointer index of oculomotor change-the difference between adjacent "straight ahead" and "visual target" settings-will be used for this purpose. As noted in the Results section, the two measures of oculomotor change are highly correlated and do not differ significantly.

The results of our experiment can now be described in terms of the two kinds of adaptive change, using the mean data for $10 \mathrm{Ss}$. At the start of the experiment, $S$ saw the visual target (through the prism) $7.9 \mathrm{~cm}$ to left of center, and he set the pointer more or less accurately underneath that point. By the 15th trial, oculomotor adaptation had caused the visual target to appear only $2.6 \mathrm{~cm}$ to left of center. However, in attempting to set the pointer at $2.6 \mathrm{~cm}, \mathrm{~S}$ was unsuccessful: the proprioceptive shift caused him to set the pointer $1.5 \mathrm{~cm}$ to the right of where he was trying to set it, and the result was a pointer setting of $1.1 \mathrm{~cm}$ to left of center.

\section{DISCUSSIOH}

Oculomotor change as a type of eye movement. Consider a $\mathrm{S}$ who continues to look straight ahead while achieving complete oculomotor adaptation. (Assume a visual target which is veridically straight ahead and a prism which displaces it, say, 14 degrees toward S's left.) Before any adaptation has taken place, we find $S$ looking straight ahead more or less accuratelyi.e., 14 degrees to right of the target. However, after the adaptive process has gone to completion, we find him looking directly at the target, which now appears to him to be straight ahead. In other words, during the course of adaptation, the anterior portion of his eye has rotated 14 degrees toward his left; and, simultaneously, the target has appeared to move 14 degrees toward his right.

This 14 degree ocular rotation is involuntary, and $S$ is not aware of it. An experimenter, noting the deviation of gaze, might repeat the instruction, "Look straight ahead." The S's reply would be, "I am looking straight ahead"; and he continues to direct his gaze 14 degrees to the left of his head-body straight ahead.

The oculomotor change in prism adaptation thus has the essential characteristics of the eye movements which Dodge (1903) classified as Type V, and which today are called "vergence" movements: slow, involuntary ocular rotations accompanied by apparent movement of the visual environment, the direction of apparent movement being opposite to the direction of rotation of the anterior portion of the eye.

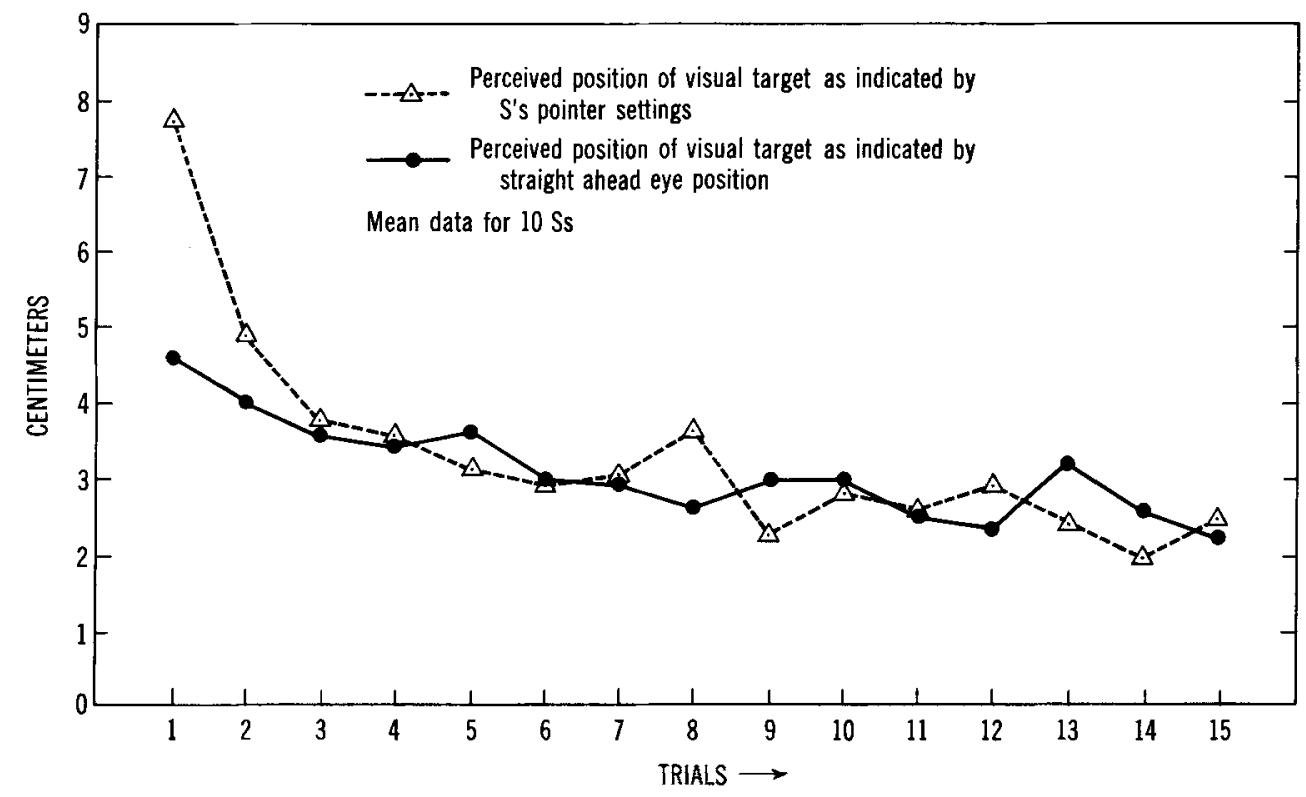

Fig. 2. Showing the close correspondence between two independent measures of oculomotor change. The pointer setting measure is the disparity between adjacent "straight ahead" and "visual target" settings. The eye position measure is the numerical difference between the prism deviation and "straight ahead" eye position. Each represents S's estimate of how far the visual target appears displaced from straight ahead. 


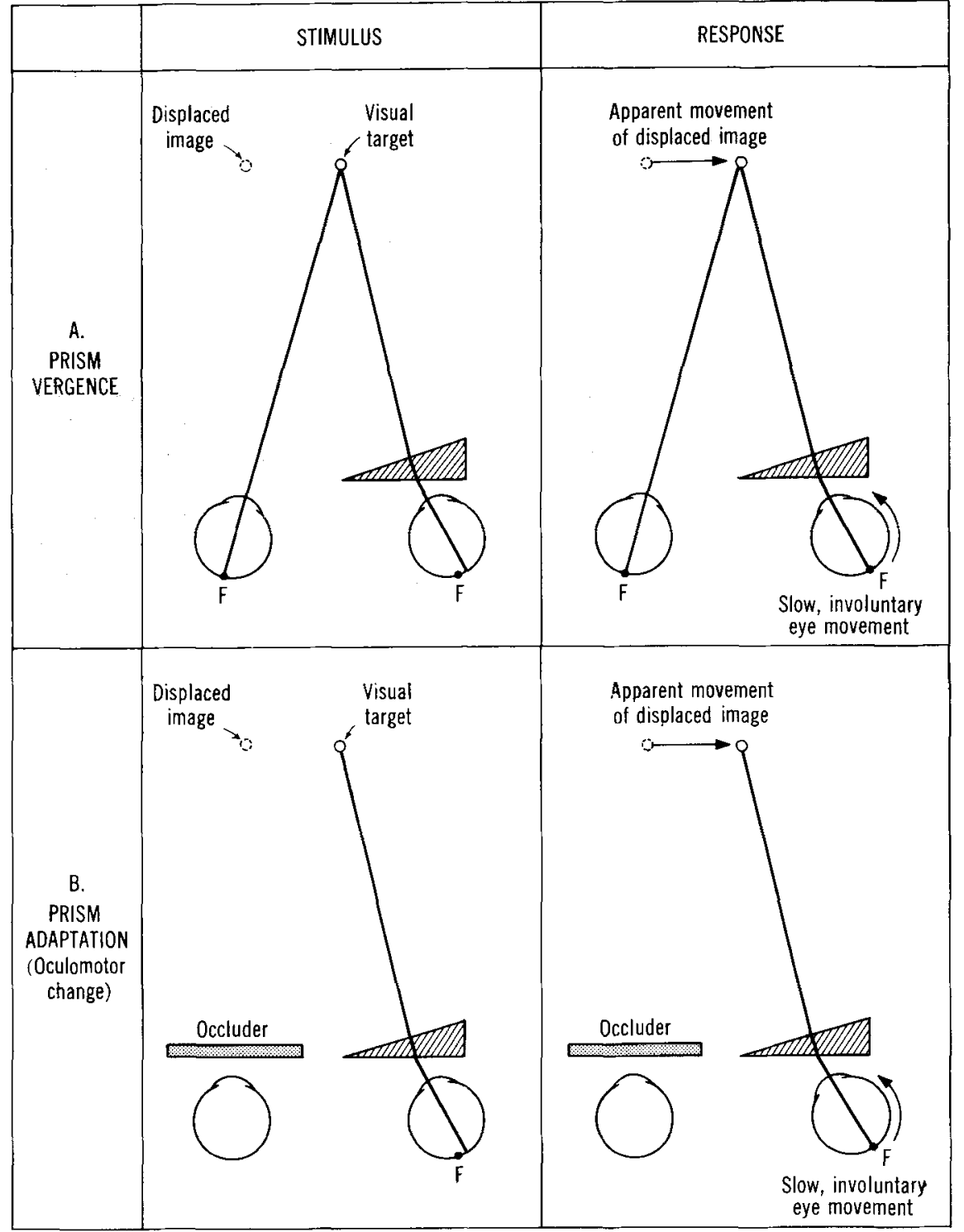

Fig. 3. The analogy between prism adaptation (monocular) and prism vergence (binocular). The symbol " $F$ " indicates the fovea centralis. In prism adaptation, the position of the left eye (behind the occluder is indeterminate.
Analogy between prism adaptation and prism vergence. Figure 1A illustrates the type of eye movement known as "prism vergence," which subserves the recovery of single binocular vision when fusion has been temporarily disrupted by placing a prism before one eye (Alpern, 1962). The eye behind the prism rotates to recover fusion; and this rotation (slow and involuntary) is accompanied by apparent movement of the visual target as seen from that eye. (As a clinical test for the strength of binocular reflexes, the prism vergence procedure is often carried out with the total amount of prism divided equally between the two eyes; in Fig. 1, however, in order to illustrate the analogy to prism adaptation (Fig. 1B), only one prism is used.)

It is clear from Fig. 1 that prism vergence and the oculomotor type of prism adaptation are similar re- sponses to different stimuli. In prism vergence, the oculomotor change is brought about by the tendency toward the recovery of single binocular vision. In prism adaptation, on the other hand, the stimulus conditions which produce an oculomotor change have to do with the geometric properties of the visual display seen through prisms (McLaughlin, Rifkin, \& Webster, 1966).

Parametric adjustment. It has been suggested that the behavioral changes which occur during adaptation to displaced vision can best be described in terms of von Holst's distinction between reafferent and exafferent stimulation (Held \& Bossom, 1961; von Holst, 1954). Our primary concern here, however, is not with the stimulus conditions which favor adaptation, but with the response mechanism; and, specifically, with the mechanism of the oculomotor adaptive response. 


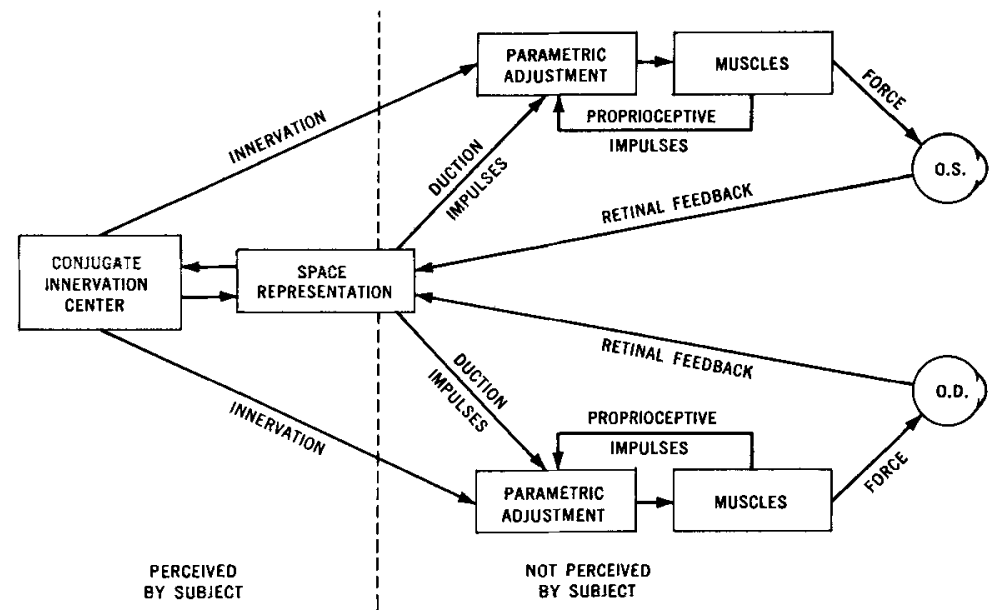

Fig. 4. Ludvigh's diagram for the qualitative explanation of certain oculomotor phenomena, particularly those in which parametric adjustment (see text) plays a prominent role. The diagram has been taken intact from Ludvigh (1952). The efferent impulses for involuntary ocular rotations (such as those involved in prism adaptation) are represented in the diagram by "duction impulses". Note that "proprioceptive impulses" remain to right of the dotted line and therefore do not provide conscious awareness of eye position.

More appropriate for our purposes than von Holst's theory are several other models of the oculomotor control system which have been advanced in recent years (Ludvigh, 1952; MacKay, 1962; Young \& Stark, 1963; Fender, 1964). Of these, Ludvigh's "parametric adjustment" theory has the advantage that it is specifically concerned with the kind of change which, in our view, occurs during oculomotor adaptation to prisms.

Ludvigh pointed out that in everyday use of the eyes, the amount of ocular rotation which results from a given pattern of innervation to the extra-ocular muscles is altered by variations in such parameters as tonus of the individual muscles, position of the eye, and metabolic state of the organism. He suggested that, in the presence of these parametric fluctuations, the system might retain the capacity for precise control by means of a sub-system (the parametric adjustment center) which is sensitive to parametric fluctuations. According to Ludvigh's model (Fig. 4), efferent innervation to the extra-ocular muscles passes through the parametric adjustment center, where it is modified (according to the momentary values of the various parameters) in such a way that a given pattern of voluntary innervation always produces the sameangular rotation of the eye.

To illustrate the relevance of this theory to prism adaptation, consider the $\mathrm{S}$ who, before adapting to prisms, held his eyes reasonably well centered when he looked straight ahead; but who, after adapting, responded to the instruction "look straight ahead" by directing his gaze some 14 degrees to right of center. According to Ludvigh's model, the same innervation was sent from the space representation center in both instances. Before adaptation, the efferent innervation passed through the parametric adjustment center without substantial modification. After adaptation, however, the same pattern of innervation was considerably modified at the parametric adjustment center, and the result was a markedly different eye position. According to this interpretation, the parametric adjustment center is the locus of the oculomotor change in prism adaptation.

Ordinarily, the parametric adjustment is made much more slowly in prism adaptation than in prism vergence; but this may not always be the case. In our laboratory, we have occasionally observed that some highly practiced Ss adapt almost instantly when the prism goggles are put on, before any measurements of reaching behavior can be made; and Taylor (1962) has descrited a $\mathrm{S}$ who became able to ride a bicycle while repeatedly donning and removing the prisms. It may be that such Ss have learned to make a prismadaptive parametric adjustment with a speed and precision which, for most Ss, is possible only in vergence movements. Using highly practiced Ss, it should eventually prove possible to demonstrate a prismadaptive eye movement having the reaction-time and velocity characteristics of a prism vergence movement.

\section{References}

Alpem, M. Types of movement. In H. Davson (Ed.), The eye. New York and London: Academic Press, 1962. Ch. 5, p. 91ff.

Dodge, R. Five types of eye movement in the horizontal meridian plane of the field of regard, Amer. J. Physiol., 1903, 8, 307$329 ;$ p. 327.

Fender, D. H. The eye-movement control system: evolution of a model. In R. F. Reiss (Ed.), Neural theory and modeling. Starford, Calif.: Stanford University Press, 1964. Ch. 15.

Hamilton, C. R. Intermanual transfer of adaptation to prisms. Amer. J. Psychol., 1964, 77, 457-462.

Harris, C. S. Adaptation to displaced vision: visual, motor, or proprioceptive change? Science, 1963, 140, 812-813.

Harris, C. S. Perceptual adaptation to inverted, reversed, and displaced vision. Psychol. Rev., 1965, 72, 419-444.

Hay, J. C., \& Pick, H. L., Jr. Visual and proprioceptive adaptation to optical displacement of the visual stimulus. J. exp. Psychol., $1966,71,150-158$.

Held, R., \& Bossom, J. Neonatal deprivation and adult rearrangement: complementary techniques for analyzing plastic sensorymotor coordinations. J. comp. physiol. Psychol., 1961, 54, 33-37. von Helmholtz, H. Handbuch der physiologischen optik. Leipzig: Leopold Voss, 1867. 
von Holst, E. Relations between the central nervous system and the peripheral organs. Brit. J. anim. Behav., 1954, 2, 89-94.

Kalil, R. E., \& Freedman, S. J. Persistence of ocular rotation following compensation for displaced vision. Percept. mot. Skills, 1966, 22, 135-139.

Ludvigh, E. Control of ocular movements and visual interpretation of the environment. Arch. Ophthal., 1952, 48, 442-448.

Mackay, D. M. In C. A. Muses (Ed.), Aspects of the theory of artificial intelligence. New York: Plenum Press, 1962. P. 83-103.

McLaughlin, S. C., \& Bower, J. L. Selective intermanual transfer of adaptive effects during adaptation to prism. Psychon. Sci. $1965,2,69-70$.

McLaughlin, S. C., Rifkin, K. I., \& Webster, R. G. Oculomotor adaptation to wedge prisms with no part of the body seen. Percept. \& Psychophys., 1966, 1, 452-458.

Merton, P. A. Absence of conscious position sense in the human eye. In M. Bender (Ed.), The oculomotor system. New York: Harper \& Row, 1964. Ch. 14.

Taylor, J. G. The behavioral basis of perception. New Haven and London: Yale University Press, 1962. P. 204.
Young, L. R., \& Stark, L. Variable feedback experiments testing a sampled data model for eye tracking movements. I. E. E. E. Trans. Human Factors Electron., 1963, 4, 38-51.

\section{Notes}

1. 'The first edition of Helmholtz' Handbuch is cited here to give an accurate date. The quotations are from J. P. C. Southall's translation of the third edition (The Optical Society of America (1924). The description of prism adaptation in the third edition ( $1910 ; 1924$, p. $246 \mathrm{ff}$.) does not differ from that in the first edition (p. 601 ff.)

2. The phrase "judgment of the direction of the gaze" is Southall's translation of "die Beurtheilung der Blickrichtung".

3. The research described here was supported in part by Contract MD-2714 with the U. S. Army Medical Research and Development Command, and in part by Research Grant GB-4196 from the National Science Foundation.

4. The junior author is now at Sandia Corporation, Albuquerque, New Mexico.

(Accepted for publication November $7,1966$. ) 